\title{
User Preferences Related to Multimedia Elements of a Mobile Application to Prevent Diabetes
}

\author{
Yisel Pinillos-Patiño ${ }^{1}$, Yaneth Herazo-Beltrán ${ }^{1}$, Orlando Rodríguez-Cordero ${ }^{1}$, Amada Escorcia-Bermejo ${ }^{1}$, \\ Enrique Martelo-López ${ }^{2}$, José Armando Vidarte-Claros ${ }^{3}$, José Hoover Vanegas García ${ }^{4}$, \\ Gabriela Y. Cortés Moreno ${ }^{5}$ \\ ${ }^{1}$ Department of Health Sciences, Simón Bolivar University, Barranquilla, Colombia \\ ${ }^{2}$ Department of Engineering, Simón Bolívar University, Barranquilla, Colombia \\ ${ }^{3}$ Department of Health, Autonomous University of Manizales, Manizales, Colombia \\ ${ }^{4}$ School of Social and Business Studies, Autonomous University of Manizales, Manizales, Colombia \\ ${ }^{5}$ General Directorate of Quality and Health Education, Health Secretary, Mexico City, Mexico
}

Objectives: To understand user preferences related to the characteristics of an application that promotes and provides education on healthy habits to correctly design multimedia elements. Methods: We conducted a comprehensive qualitative study with a hermeneutical strategy, which gathered information using well-researched questions that were posed to focus groups consisting of 32 participants. These participants were asked for opinions related to multimedia elements to display educational messages about physical activity and healthy eating in a mobile application. There were three analysis categories of multimedia elements: text, visual elements, and audio elements. Results: The majority of the participants, $93.75 \%$, were in the low socioeconomic stratum; $68.75 \%$ are in a civil union with their partner; $53.12 \%$ completed or failed to complete secondary school, and $68.75 \%$ were housewives. Based on the qualitative results, we found that mobile applications become mediating tools that support the adoption of actions that tend to improve lifestyles and increase knowledge about proper nutrition and physical activity. Text messages used in mobile applications should promote healthy habits and remind users of their benefits. Images and videos should be accompanied by text and audio to provide greater clarity regarding recommendations of healthy habits. Conclusions: Technology must provide accessibility and coverage opportunities, while meeting the needs and expectations of users. It should facilitate primary health intervention through education to transform unhealthy behaviors and generate lifestyles that improve the health of the user and their family context.

Keywords: Healthy Lifestyle, Proper Nutrition, Exercise, mHealth

Submitted: April 15, 2020, Revised: July 30, 2020, Accepted: August 26, 2020

\section{Corresponding Author}

Yisel Pinillos-Patiño

Department of Health Sciences, Simón Bolivar University, Carrera 59 No. 59-65, Barranquilla, Colombia. Tel: +57-3012926368, E-mail: yiselpinillos@gmail.com (https://orcid.org/0000-0001-5047-3883)

This is an Open Access article distributed under the terms of the Creative Commons Attribution Non-Commercial License (http://creativecommons.org/licenses/by-nc/4.0/) which permits unrestricted non-commercial use, distribution, and reproduction in any medium, provided the original work is properly cited.

(C) 2020 The Korean Society of Medical Informatics 


\section{Introduction}

Chronic non-communicable diseases (NCDs), are the leading causes of $63 \%$ of deaths worldwide and $86 \%$ deaths in low- and middle-income countries [1]. Type-2 diabetes mellitus (T2DM) causes 1.6 million deaths worldwide [2]. Furthermore, $9.6 \%$ of the population in North America and the Caribbean is affected by it. In Central and South America, the affected population is $8.2 \%$ [3]. In Colombia, T2DM occurs in $7 \%$ to $9 \%$ of the population aged more than 20 years, and among the causes for its frequency in women is the previous diagnosis of gestational diabetes mellitus (GDM) in association with unhealthy life habits postpartum [4-7]. According to some studies, $30 \%$ of women with GDM continue experiencing glucose alterations immediately after delivery and have a risk of $9.5 \%$ to $37 \%$ of developing T2DM [8-10].

Postpartum follow-up of women with a history of GDM is essential due to their high risk of developing T2DM, a risk that increases during the first 10 years if there are no changes in the health-related lifestyle; therefore, it is necessary to educate and advise on a healthy lifestyle based on weight control, diet, and physical exercise [11-13].

The use of information technology (IT) for health education and as a complement to the usual interventions, expands the knowledge and development of healthy selfmanagement habits, which related to multimedia elements, maintains user attention and improves the effectiveness of health education [14-16]. There are factors that determine whether multimedia elements are effective in maintaining a person's attention and achieving the proposed objectives, such as user preferences regarding the weight of texts, the length of instructions, and the type of images [17]. Previous studies have shown the acceptance of mobile applications by health service providers for the management and prevention of GDM and the associated risk factors, achieving self-control and patient compliance with glycemic monitoring and treatment. Regarding the multimedia elements used to present educational content [18], previous research did not include users of technological devices. The study by Polinski et al. [19] took into account the satisfaction and preference of patients to receive healthcare through telehealth compared to a traditional office visit, although they did not expand on the multimedia elements used to present educational content, which was the main purpose of the present study. In the Baby Steps program, a qualitative study was made on the experience of a group of women with a diagnosis of GDM during pregnancy to assess their satisfaction with the program; the findings were used to adjust the content or pre- sentation modes of the program [20]. The high prevalence of T2DM in women with previous GDM was our main motivation to design a mobile application that could contribute to healthy lifestyles for this specific population. The purpose was to explore the motivations for healthy lifestyles and the preferences of the multimedia elements used to present educational content about physical activity and healthy eating in a mobile application.

\section{Methods}

Based on a qualitative approach, a comprehensive study with a hermeneutical strategy was carried out. This study collected participants' opinions on multimedia elements used to display educational content in a mobile application, within the framework of the project. Through their responses, using a qualitative methodology, it was possible to identify their preferences regarding a mobile healthcare application. In addition, we were able to collect information on the motivations that would drive changes in their harmful habits and promote healthy lifestyles for them and their families.

The main topics addressed in this research were physical activity and proper nutrition. Based on these, three categories of analysis of the multimedia elements in the mobile application were defined. With regard to the text of the contents disclosed by the application, the questions revolved around its length and the preference between audio and text. Concerning the audio and video presentation of content, we asked the participants about their preferences regarding colors, speed of presentation, sound quality, and scenarios for physical exercise, as well as their preferences for the demonstration of physical exercises by means of graphic representations, for example, using avatars or real people (Table 1).

A non-probabilistic sample was taken from the neighborhoods of five localities (Riomar, North-Historic Center, South West, Metropolitan, and South East) into which the city of Barranquilla, Colombia is politically and administratively organized (Table 2). Through an intentional nonprobability approach, some volunteers were recruited who, in turn, attracted others, bringing 50 women together to participate in the study with the aim of recruiting participants in each of the three focus groups. Finally, the participation of 32 women with a maximum of one year postpartum and with a previous diagnosis of GDM was secured. The first focus group included 10 women, and the second and third focus groups included 11 women each. Women in the postpartum stage with a previous diagnosis of gestational diabetes were selected, since until before this project there 
Table 1. Categories and subcategories used to identify the preferences, recommendations, and characteristics of the application's multimedia elements

\begin{tabular}{cll}
\hline Category & Subcategory & \multicolumn{1}{c}{ Description } \\
\hline Preferences of the multimedia elements & Texts & Motivations to carry out healthy activities \\
& & Mobile application support to have a healthy lifestyle
\end{tabular}

Table 2. Distribution of participants according to their hometown

\begin{tabular}{lc}
\hline \multicolumn{1}{c}{ Locality } & Frequency (\%) \\
\hline Metropolitan & $6(18.75)$ \\
North Historical Center & $5(15.62)$ \\
South West & $5(15.62)$ \\
South East & $16(50.00)$ \\
\hline
\end{tabular}

was no periodic medical control on the health condition of this population, while in pregnant women it was controlled through periodic medical visits that are specific to their state; therefore, this process became a response to the need care of the postpartum population within the framework of the New Life Generation Program, supported by the Ministry of Health and the World Diabetes Foundation.

To collect information, the focus groups were maintained until information saturation was reached; the meetings were held in Points of Timely Health Care (PASOS, for its Spanish acronym) and in the Centers for Timely Comprehensive Medical Care (CAMINOS, for its Spanish acronym), near the neighborhoods where the women resided. Previously, a home visit was made to women who met the criteria to explain the purpose of the study, invite them to form the focus groups, and arrange the dates and times of the meetings.

Each meeting lasted between 90 and 120 minutes and was facilitated by a moderator and three assistants with training in physiotherapy, nutrition, systems engineering, and psychology. Three experts in qualitative research codified the information collected and created nodes, which allowed the references to be organized to reflect the data and for the preferences expressed by the participants to be compared. For this purpose, the subcategories described in Table 1 were used. Cases were created to which the nodes were assigned in which the information collected was coded, and from there, the analysis and comparison of the preferences expressed by the women was carried out.
A structured question guide was drafted based on the appropriate literature. The questions originated from the requirements that had to be collected so that the team of system engineers could design the mobile application for the educational process. First, the motivations of the participants to take care of their health and learn healthy lifestyles were investigated. Then they were asked for their opinions about using a mobile application for the educational process and which scenarios they preferred to watch the guide videos of physical activity, the contents of the motivational messages. Finally, the potential use of the application was assessed. The questions were reviewed and tested by the research team and validated with five people, which allowed for the adaptation of the language in which the guiding questions were structured. This was done for the purposes of obtaining adequate information through interviews and guaranteeing the psychometric properties of the question guide. The focus groups guide contained the introductory and opening questions of the meetings (Table 3 ).

The data obtained from audio recordings of the meetings were transcribed textually, systematically, and anonymously into Microsoft Word through the program SpeechNotesdictation notepad, a free online program to convert audio to text. The QUAGOL guide facilitated the preparation for coding and analysis of the qualitative interview data. The statistical program SPSS Statistics version 24 (IBM, Armonk, NY, USA) was used to define the frequencies of the sociodemographic characteristics, and the software NVivo 11 (QSR International, Melbourne, Australia) used for the qualitative analysis, was licensed by the Simón Bolívar University of Colombia.

Ethical considerations: The qualitative data obtained in this study was part of a macro project called Effects of an educational program mediated by mobile technologies to promote healthy lifestyles in post-pregnancy women with a previous diagnosis of gestational diabetes". The formulation of the qualitative study was based on response to aspects that 
Table 3. Guiding questions from the focus groups

\begin{tabular}{ll}
\hline \multicolumn{1}{c}{ Type } & \multicolumn{1}{c}{ Question } \\
\hline Introductory & What motivates you to keep healthy habits in your life? \\
In what way does eating well benefit your health? & What is your opinion about the support that a mobile application offers to have a healthy lifestyle? \\
Exploratory & What are the scenarios where you would most like to do physical activity? \\
& Would you like to receive instructions on how to do physical exercises in text or audio messages? \\
& Would you like to receive recommendations for healthy eating and physical activity in the form of text, video, \\
& How would you like the pictures and videos of the application to be? \\
& Would you like to see real people or avatars in the videos? \\
What characteristics should the audios contained in the application have? & How does technology help educate people to have healthy lifestyles? \\
Closing & Would you use a free application that is installed on your cell phone?
\end{tabular}

refer to behavior change for healthy lifestyles according to the Prochaska transtheoretical model [21]. The participants were required to sign an informed consent document. The study was approved by the ethics committee of the Simón Bolívar University. The study was classified as a risk-free investigation in accordance with Resolution 8340 of the Ministry of Health of Colombia.

\section{Results}

The study included 32 postpartum female participants, after a maximum of 1 year postpartum, who were part of the New Life Generation Program led by Fundación Vida Nueva. The average age was $27 \pm 4.87$ years. Most of the participants (50\%) came from the South East neighborhood, followed by those who resided in the metropolitan neighborhood.

Regarding the sociodemographic characteristics of the participants, 93.75\% belonged to a low socioeconomic status, and $68.75 \%$ of the women lived in a consensual union with their partners. Regarding educational level, 53.12\% reported having attended high school (complete or incomplete education), followed by $28.12 \%$ who reported having completed their technical or technological training. Regarding occupation, $68.75 \%$ were housewives, followed by $18.75 \%$ who indicated that they work independently (Table 4).

The analysis of the results of the focus groups on the multimedia element categories (texts, pictures, videos, and audio) led to the following findings.

(1) Motivation to carry out healthy activities: The women considered that commitment and dedication are necessary for changes in health-related lifestyles to have a healthy fu- ture and a better life. Members of the study group said that their main motivation for achieving positive changes in their daily lives and improving their habits was their children.

Children as the central axis of change:

"My children motivate me to get ahead..." (Focus

Group 1 Female 2)

Moreover, they were also motivated by their personal appearance, given that a healthy physical appearance would give them self-confidence because they would feel good about their bodies and themselves with their family, as stated.

\section{(2) Personal appearance}

"Well...the truth is that looking good physically motivates me... also to be healthy." (Focus Group 2 Female 1)

From the nutritional point of view, they indicated that having good eating habits helps them look physically well and prevent diseases. They were aware that if they became sick they would have no one to take care of their children, that the combination of eating healthy and exercising would benefit them because it would help them maintain a healthy weight and the energy to perform daily activities.

\section{(3) Having good eating habits to prevent diseases}

“...I got really fat-I ended up weighing ninety kilos, and I am motivated by my health and my children, because one has to think about the children." (Focus Group 3 Female 5)

(4) Support of mobile applications to have a healthy lifestyle: Mobile applications can encourage people to improve their lifestyles, increase knowledge about proper nutrition, and learn to be more physically active, due to the permanent 
Table 4. Sociodemographic characteristics of the participants $(n=32)$

\begin{tabular}{lc}
\multicolumn{1}{c}{ Characteristic } & Value \\
\hline Age (yr) & $27 \pm 4.87$ \\
Socioeconomic level & \\
Level 1 (low-low socioeconomic level) & $30(93.75)$ \\
Level 2 (low socioeconomic level) & $2(6.25)$ \\
Civil status & \\
Single & $5(15.62)$ \\
Married & $3(9.37)$ \\
Consensual union & $22(68.75)$ \\
Separated/divorced & $2(6.25)$ \\
Educational level & \\
None or incomplete primary school & $3(9.37)$ \\
Complete primary school & $2(6.25)$ \\
Complete or incomplete secondary school & $17(53.12)$ \\
Technical or technological training & $9(28.12)$ \\
University studies & $1(3.12)$ \\
Occupation & $22(68.75)$ \\
Self-employed work & $2(6.25)$ \\
Subordinate employment & $6(18.75)$ \\
Housewife & $2(6.25)$ \\
Student & \\
\hline
\end{tabular}

Values are presented as mean \pm standard deviation or frequency (\%).

reminder to maintain proper and healthy habits, and to keep track of practices performed that benefit their health.

They indicated that mobile applications contributed to their healthy lifestyles because they could easily access them from their cell phones, breaking one of the barriers to physical exercise, which is the lack of resources to go to physical activity environments which are not free. In addition, it would allow for the use of a variety of elements in various places where physical activity is performed, such as the home, parks, and sports venues close to their homes, and as a means of transportation, in addition to the provision of recommendations to eat a healthy diet through the effective use of available resources.

(5) Use of free mobile applications installed on the user's mobile phone: It was found that the use of an application that does not require permanent connection to the Internet attracted their attention, given that not all participants had access to mobile phone plans to check on the Internet on a daily basis. Besides, they also did not always have free $\mathrm{Wi}-\mathrm{Fi}$ or financial resources to buy data to access Internet media to make inquiries about health issues.

(6) Texts of multimedia elements: Is very important that the messages carry a motivational content, stimulate the practice of healthy habits, and remind the user of the benefits of adopting them. Similarly, the participants affirmed that text messages should be informative and include accurate and clear indications. Short sentences are easier to remember and encourage the user. They suggested that the instructions given to carry out exercises and the preparation of food should be clear and precise.

(7) Visual elements (pictures and videos): Pictures and videos should be accompanied by text and audio as a complement to what is seen and heard. Thus, further clarification is provided with the recommendations for the proper practice of healthy habits. Regarding pictures, they suggested the use of bright colors as well as the inclusion of icons and pictures showing real people. They preferred texts, pictures, videos, and audios be used when presenting content through the mobile application.

In addition to pictures, they suggested using videos that show the correct way to execute the recommended physical exercise, preferably with people and not avatars. They emphasized that if the people in the videos demonstrating the physical exercise plan were not models or actors, it would give the mobile application a more real presence, adjusted to their daily life.

They recommended that the videos be filmed in everyday places, realistic for the context in which the participants perform their activities (home, parks, community), without losing the objective of a quiet scenario and the use of available resources or, alternatively, in reach of people with low incomes.

(8) Elements of Audios: They recommend a moderate speed so that they would be able to visualize and understand what was being demonstrated. They highlighted that the guidelines should be specified through voice messages.

\section{Discussion}

As stated above, all of the participants had GDM during their pregnancies, and they were very young women of a low socioeconomic level. These characteristics place them in a population at risk for unhealthy lifestyles and a greater probability of suffering from NCDs; therefore, there is a need to implement accessible and attractive health interventions for a young population. The study included diversity of the sample in terms of age, and it allowed us to clarify the potential role of technology to support a healthy postpartum 
lifestyle in young women and young adults. There has been little research that has provided a qualitative description of people's preferences regarding the characteristics of applications designed to promote their health and well-being.

The time between childbirth and the interviews varied among the participants; some of them had given birth more recently than others (almost 1 year). Nevertheless, we studied postpartum women for a maximum period of 1 year postpartum because it is a key time to intercede in the behaviors that have been fostered before childbirth. The findings of the study lack generalizability; they are only applicable to people with characteristics similar to those included in our study. The study included only women, which limits the knowledge of the preferences and perspectives of men.

With regard to the motivations and advantages of using a mobile application, the results agree with the findings of Lau et al. [22], who reported that the majority of women participating in their study expressed the practicality and ease of access anytime and anywhere, according to their status and their roles, the recommendation of friendly activities adapted to their needs and expectations as the main advantages of using mobile applications [23]. Stahl et al. [24] showed that mobile applications are an option for the achievement of healthy lifestyles at all ages. In that study, older people expressed the benefits of using these types of technologies in terms of organizing their life habits, promoting self-care, learning to prepare healthy foods, and especially, practicing good behaviors. According to the authors of [22], the use these tools to create programs for correct health education increased; the apps represent a social advance, and an advantage in the world of health.

The study conducted by Lau et al. [22] corresponds with the findings of the present study; the participants indicated that the platform should be easily accessible because it would stimulate and promote its more frequent use. Regarding aesthetic appeal, they considered it a determining factor in the use of the application.

Regarding the characteristics displayed, they stated that these improve the ability of women to understand their progress and acknowledge their food intake. Therefore, the visual and graphic elements must be considered in the development of an application. Furthermore, the option of using a variety multimedia elements in the intervention (short video sequences, graphs, photos, web-based questionnaires, and animation) would make an application more attractive. Therefore, the visual and graphic elements must be considered when developing an application to make it more attractive [22].
McMillan et al. [25] referred to a real interface that encourages the use of technology, which implies the speed and ease of use of the application. Brandt et al. [26] reported that most people value the ability to measure their progress and recognize their effort, which is consistent with what was expressed by the women in this study, who said that they should be able to see the progress of their tasks as they perform them. This also corresponds to the findings of McMillan et al. [25], who analyzed the various ways in which technology allows users to manage their own health, in addition to having objectives to achieve, reminders of activities to be carried out, and a reward system that encourages them to set limits on their calorie consumption. The participants in that study reported that receiving comments was a positive experience.

Dallinga et al. [27] consulted experts in technology and health sciences who emphasized that a feedback option, as well as widespread adaptation, should be integrated into an application; they suggested several feedback options, such as motivational and educational feedback. Another key element of adaptation is the flexibility of the application, that is, the ability to adjust the application and the adaptability of the application. The experts considered that people would be more willing to download an application if it was free.

In conclusion, for the design of an effective mobile application for health education, clarity is required on the fundamental needs, tastes, and preferences of potential users. The features of the technological tool should be designed in such a way that its use is motivating, characteristics related to the use of visual and auditory elements should be carefully considered in terms of the shape of their design. Preferably, texts with clear and precise information should be used. Images and videos must be accompanied by text and audio as a complement to what is seen and heard. The use of bright colors and the inclusion of icons and images that show real people is preferred. Demonstrations of physical exercises should be presented through videos of people in common places, such as home and parks.

\section{Conflict of Interest}

No potential conflict of interest relevant to this article was reported.

\section{Acknowledgments}

The authors thank all the women who are part of the New Life Generation Program; the field work team of Fundación 
Vida Nueva, for their contribution regarding the collection of information. It was also supported by the Scientific Committee of the New Life Generation Program of the Mayor's Office of Barranquilla. The Administrative Department of Science, Technology, and Innovation (Colciencias) in Colombia, by the funding to conduct the research in call 715 of 2015. And the Simón Bolívar University for the institutional support and backing.

\section{ORCID}

Yisel Pinillos-Patiño (https://orcid.org/0000-0001-50473883)

Yaneth Herazo-Beltrán (https://orcid.org/0000-0003-37524353)

Orlando Rodríguez-Cordero (https://orcid.org/0000-00023592-062X)

Amada Escorcia-Bermejo (https://orcid.org/0000-00022939-8433)

Enrique Martelo-López (https://orcid.org/0000-0003-27185853)

José Armando Vidarte-Claros (https://orcid.org/0000-00027982-3848)

José Hoover Vanegas García (https://orcid.org/0000-00021424-7990)

Gabriela Y. Cortés Moreno (https://orcid.org/0000-00024506-8223)

\section{References}

1. World Health Organization. Global action plan for the prevention and control of NCDs 2013-2020 [Internet]. Geneva, Switzerland: World Health Organization; 2013 [cited at 2020 Sep 15]. Available from: https://www.who. int/nmh/events/ncd_action_plan/en/.

2. World Health Organization. Global report on diabetes [Internet]. Geneva, Switzerland: World Health Organization; 2016 [cited at 2020 Sep 15]. Available from: https://www.who.int/diabetes/global-report/en/.

3. Kaiser Family Foundation. The U.S. government and global non-communicable disease efforts [Internet]. San Francisco (CA): Kaiser Family Foundation; 2019 [cited at $2020 \mathrm{Sep} \mathrm{15]}$. Available from: https://www.kff.org/ global-health-policy/fact-sheet/the-u-s-governmentand-global-non-communicable-diseases/.

4. Ministry of Health and Social Protection of Colombia. Clinical Practice Guide for the diagnosis, treatment and follow-up of type 2 diabetes mellitus in the popula- tion over 18 years of age [Internet]. Bogota, Colombia: Ministry of Health and Social Protection; 2016 [cited at 2020 Sep 15]. Available from: http://gpc.minsalud.gov. co/gpc_sites/Repositorio/Conv_637/GPC_diabetes/ DIABETES_TIPO_2_COMPLETA.pdf.

5. Aguilar Cordero MJ, Sanchez Lopez AM, Rodriguez Blanque R, Noack Segovia JP, Pozo Cano MD, LopezContreras G, et al. Physical activity by pregnant women and its influence on maternal and foetal parameters: a systematic review. Nutr Hosp 2014;30(4):719-26.

6. Egan AM, Vellinga A, Harreiter J, Simmons D, Desoye G, Corcoy R, et al. Epidemiology of gestational diabetes mellitus according to IADPSG/WHO 2013 criteria among obese pregnant women in Europe. Diabetologia 2017;60(10):1913-21.

7. Pinillos-Patino Y, Herazo-Beltran Y, Mendoza-Charris H, Kuzmar I, Galeano-Munoz L. Relationship between physical activity in pregnant and gestational diabetes: cross-sectional study. Revista Latinoamericana de Hipertensión 2017;12(5):138-43.

8. Tobias DK, Hu FB, Chavarro J, Rosner B, Mozaffarian D, Zhang C. Healthful dietary patterns and type 2 diabetes mellitus risk among women with a history of gestational diabetes mellitus. Arch Intern Med 2012;172(20):156672.

9. Kim C, Newton KM, Knopp RH. Gestational diabetes and the incidence of type 2 diabetes: a systematic review. Diabetes Care 2002;25(10):1862-8.

10. Lo JC, Yang J, Gunderson EP, Hararah MK, Gonzalez JR, Ferrara A. Risk of type 2 diabetes mellitus following gestational diabetes pregnancy in women with polycystic ovary syndrome. J Diabetes Res 2017;2017:5250162.

11. Kim C. Maternal outcomes and follow-up after gestational diabetes mellitus. Diabet Med 2014;31(3):292301.

12. Heatley E, Middleton P, Hague W, Crowther C. The DIAMIND study: postpartum SMS reminders to women who have had gestational diabetes mellitus to test for type 2 diabetes: a randomised controlled trial: study protocol. BMC Pregnancy Childbirth 2013;13:92.

13. Tierney M, O'Dea A, Danyliv A, Noctor E, McGuire B, Glynn L, et al. Factors influencing lifestyle behaviours during and after a gestational diabetes mellitus pregnancy. Health Psychol Behav Med 2015;3(1):204-16.

14. Kaufman N, Khurana I. Using digital health technology to prevent and treat diabetes. Diabetes Technol Ther 2016;18 Suppl 1(Suppl 1):S56-68.

15. Harrison S, Stadler M, Ismail K, Amiel S, Herrmann- 
Werner A. Are patients with diabetes mellitus satisfied with technologies used to assist with diabetes management and coping? A structured review. Diabetes Technol Ther 2014;16(11):771-83.

16. Silva BM, Rodrigues JJ, de la Torre Diez I, Lopez-Coronado M, Saleem K. Mobile-health: a review of current state in 2015. J Biomed Inform 2015;56:265-72.

17. Ranney ML, Choo EK, Cunningham RM, Spirito A, Thorsen M, Mello MJ, et al. Acceptability, language, and structure of text message-based behavioral interventions for high-risk adolescent females: a qualitative study. J Adolesc Health 2014;55(1):33-40.

18. Nikolopoulos M, Karampela I, Antonakos G, Tzortzis E, Stratigou T, Diomidous M, et al. Mobile phone applications for gestational diabetes mellitus: appraisal and perspectives. Stud Health Technol Inform 2019;262:3942.

19. Polinski JM, Barker T, Gagliano N, Sussman A, Brennan TA, Shrank WH. Patients' satisfaction with and preference for telehealth visits. J Gen Intern Med 2016;31(3):269-75.

20. Sukumar N, Dallosso H, Saravanan P, Yates T, Telling C, Shorthose K, et al. Baby Steps - a structured group education programme with accompanying mobile web application designed to promote physical activity in women with a history of gestational diabetes: study protocol for a randomised controlled trial. Trials 2018;19(1):682.

21. Herazo BY, Hernandez EJ, Dominguez AR. Stages of change and levels of physical activity in university students of Cartagena (Colombia). Salud Uninorte 2012;28(2):298-307.

22. Lau Y, Cheng LJ, Chi C, Tsai C, Ong KW, Ho-Lim SS, et al. Development of a healthy lifestyle mobile app for overweight pregnant women: qualitative study. JMIR Mhealth Uhealth 2018;6(4):e91.

23. Stahl ST, Emanuel J, Albert SM, Dew MA, Schulz R, Robbins-Welty $\mathrm{G}$, et al. Design and rationale for a technology-based healthy lifestyle intervention in older adults grieving the loss of a spouse. Contemp Clin Trials Commun 2017;8:99-105.

24. San Mauro Martin I, Gonzalez Fernandez M, Collado Yurrita L. Mobile applications for nutrition, dietetics and healthy habits; analysis and consequences of an increasing trend. Nutr Hosp 2014;30(1):15-24.

25. McMillan B, Easton K, Goyder E, Delaney B, Madhuvrata $\mathrm{P}$, Abdelgalil R, et al. Reducing risk of type 2 diabetes after gestational diabetes: a qualitative study to explore the potential of technology in primary care. Br J Gen Pract 2018;68(669):e260-e267.

26. Brandt CJ, Sogaard GI, Clemensen J, Sndergaard J, Nielsen JB. General practitioners' perspective on ehealth and lifestyle change: qualitative interview study. JMIR Mhealth Uhealth 2018;6(4):e88.

27. Dallinga J, Janssen M, van der Werf J, Walravens R, Vos $S$, Deutekom M. Analysis of the features important for the effectiveness of physical activity-related apps for recreational sports: expert panel approach. JMIR Mhealth Uhealth 2018;6(6):e143. 